\title{
ON THE NUMBER OF ISOLATED EIGENVALUES OF A PAIR OF PARTICLES ON THE HALF-LINE
}

\author{
JOACHIM KERNER
}

Abstract. In this note we consider a pair of particles moving on the half-line $\mathbb{R}_{+}$with the pairing induced by a hard-wall potential. This model was first introduced in [12] and later applied to investigate condensation of electron pairs in a quantum wire [11, 10]. For this, a detailed spectral analysis proved necessary and as a part of this it was shown in [10] that, in a special case, the discrete spectrum of the Hamiltonian consists of a single eigenvalue only. It is the aim of this note to prove that this is generally the case.

Mathematics subject classification (2010): 82B26, 82B10, 81V70, 81Q10, 81Q35, 82D77, 82D80, 82D55.

Keywords and phrases: Discrete spectrum, interacting particles, quantum wire.

\section{REFERENCES}

[1] J. Bardeen, L. N. Cooper, J. R. SchriefFer, Theory of superconductivity, Phys. Rev. 108, (1957), 1175-1204.

[2] J. Blank, M. HAVliČEK, P. EXNER, Hilbert space operators in quantum physics, Springer, 2008.

[3] J. Bolte, J. Kerner, Quantum graphs with singular two-particle interactions, J. Phys. A 46, (2013), 045206.

[4] J. Bolte, J. Kerner, Quantum graphs with two-particle contact interactions, J. Phys. A 46, (2013), 045207.

[5] L. N. Cooper, Bound electron pairs in a degenerate Fermi gas, Phys. Rev. 104, (1956), 1189-1190.

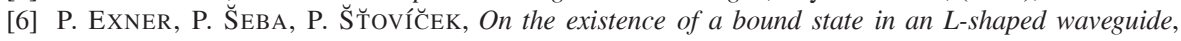
Czechoslovak Journal of Physics B , (1989), 1181-1191.

[7] T. Giamarchi, Quantum Physics in one dimension, Oxford University Press, 2004.

[8] T. Giamarchi, One-dimensional physics in the 21st century, Comptes Rendus Physique 17, (2016), 322-331.

[9] D. Gilbarg, N. S. Trudinger, Elliptic partial differential equations of second order, Springer, 1983.

[10] J. Kerner, On bound electron pairs on the half-line, Rep. Math. Phys. 83, (2019), 129-138.

[11] J. KeRner, On pairs of interacting electrons in a quantum wire, J. Math. Phys. 59, (2018), 063504.

[12] J. Kerner, T. MÜHlenbruch, On a two-particle bound system on the half-line, Rep. Math. Phys. 80, (2017), 143-151.

[13] S. NONNENMACHER, Lecture notes: Spectral theory of selfadjoint operators, (2017).

[14] F. QueISSER, W. G. UnRUh, Long-lived resonances at mirrors, Phys. Rev. D 94, (2016), 116018.

[15] S. EgGer, J. KeRner, K. PANKRASHKin, Bound states of a pair of particles on the half-line with a general interaction potential, arXiv:1812.06500, to appear in J. Spec. Theory. 